\title{
GEOMETRIC CONDITION FOR UNIVERSAL INTERPOLATION IN $\hat{\mathcal{E}}^{\prime}$
}

\author{
BY
}

WILLIAM A. SQUIRES

\begin{abstract}
It is known that if $h$ is an entire function of exponential type and $Z(h)=\left\{z_{k}\right\}_{k=1}$ with $\left|h^{\prime}\left(z_{k}\right)\right| \geqslant \varepsilon \exp \left(-c\left|z_{k}\right|\right)$ for constants $\varepsilon, C$ independent of $k$, then $\left\{z_{k}\right\}_{k=1}^{\infty}$ is a universal interpolation sequence. That is, given any sequence of complex numbers $\left\{a_{k}\right\}_{k=1}^{\infty}$ such that $\left|a_{k}\right| \leqslant A \exp \left(B\left|z_{k}\right|\right)$ for constants $A, B$ independent of $K$ then there exists $g$ of exponential type such that $g\left(z_{k}\right)=a_{k}$. This note is concerned with finding geometric conditions which make $\left\{z_{k}\right\}_{k=1}^{\infty}$ a universal interpolation sequence for various spaces of entire functions. For the space of entire functions of exponential type a necessary and sufficient condition for $\left\{z_{k}\right\}_{k=1}^{\infty}$ to be a universal interpolation sequence is that $\int_{0}^{z_{k} k} n\left(z_{k}, t\right) d t / t \leqslant C\left|z_{k}\right|+D, k=1$, $2, \ldots$, where $n\left(z_{k}, t\right)$ is the number of points of $\left\{z_{k}\right\}_{k=1}^{\infty}$ in the disc of radius $t$ about $z_{k}$, excluding $z_{k}$, and $C, D$ are constants independent of $k$. Results for the space $\hat{\mathcal{E}}^{\prime}=\left\{f\right.$ entire ||$\left.f(z) \mid \leqslant A \exp \left[B|\operatorname{Im} z|+B \log \left(1+|z|^{2}\right)\right]\right\}$ are given but the theory is not as complete as for the above example.
\end{abstract}

1. Introduction. In this note we will study the problem of finding necessary and sufficient conditions for universal interpolation in various spaces of entire functions. Our main interest will be to give geometric conditions, that is, conditions which only depend on the distribution of points of our interpolation sequence.

Let $A_{1}$ denote the space of entire functions of exponential type, that is, all entire functions $h$ satisfying

$$
|h(z)| \leqslant A \exp (B|z|), \quad z \in \mathbf{C},
$$

where $A$ and $B$ are constants depending only on $h$. A sequence $\left\{z_{k}\right\}_{k=1}^{\infty} \subset \mathbf{C}$ with $\left|z_{k}\right| \uparrow \infty$ is said to be a universal interpolation sequence for $A_{1}$ if, for all $\left\{a_{k}\right\}_{k=1}^{\infty} \subset \mathbf{C}$ such that

$$
\left|a_{k}\right| \leqslant A \exp \left(B\left|z_{k}\right|\right), \quad k=1,2, \ldots,
$$

for constants $A, B$ independent of $k$, there exists $f \in A_{1}$ such that $f\left(z_{k}\right)=a_{k}$.

A. F. Leont'ev $[\mathbf{L}]$ discovered the following theorem which illustrates the necessary and sufficient conditions for universal interpolation found previously.

ThEOREM. Given $\left\{z_{k}\right\}_{k=1}^{\infty}=Z(h)$ for $h \in A_{1}$ then $\left\{z_{k}\right\}_{k=1}^{\infty}$ is a universal interpolation sequence for $A_{1}$ if and only if

$$
\left|h^{\prime}\left(z_{k}\right)\right| \geqslant \varepsilon \exp \left(-C\left|z_{k}\right|\right), \quad k=1,2, \ldots
$$

Received by the editors August 4, 1981 and, in revised form, November 15, 1982.

1980 Mathematics Subject Classification. Primary 30D15, 30E05; Secundary 30C15, 42A38. 
In this case our necessary and sufficient geometric condition is that

$$
\int_{0}^{\left|z_{k}\right|} n\left(z_{k}, t\right) \frac{d t}{t} \leqslant C\left|z_{k}\right|+D, \quad k=1,2, \ldots,
$$

where $n\left(z_{k}, t\right)$ is the number of points of $\left\{z_{k}\right\}_{k=1}^{\infty}$ in the disc of radius $t$ about $z_{k}$, excluding $z_{k}$ and $C, D$ are independent of $k$.

The advantage of condition (4) is that given any subset $\left\{z_{k}\right\}_{k=1}^{\infty} \subset \mathbf{C}$ it is a simple calculation to determine whether $\left\{z_{k}\right\}_{k=1}^{\infty}$ is a universal interpolation sequence. For example, if $\left\{z_{k}\right\}_{k=1}^{\infty}$ is a universal interpolation sequence for $A_{1}$ then the distance between $z_{j}$ and $z_{k}$ for $j \neq k$ must be at least $\varepsilon \exp \left(-C\left|z_{k}\right|\right)$ with $\varepsilon, C$ independent of $j$, $k$. It also follows easily that there can be at most $\left(C\left|z_{k}\right|+D\right) / \log \left|z_{k}\right|$ points of $\left\{z_{k}\right\}_{k=1}^{\infty}$ in the disc of radius 1 about $z_{k}$. An example can be given to show that this density is attained.

We will also consider the question of necessary and sufficient geometric conditions for universal interpolation in spaces of entire functions defined by more general growth conditions. In particular, we will be interested in the space $\hat{\mathcal{E}}^{\prime}$, that is, the space of entire functions $f$ such that

$$
|f(z)| \leqslant A \exp (B \hat{p}(z)), \quad z \in \mathbf{C},
$$

where $\hat{p}(\mathrm{z})=|\operatorname{Im} z|+\log \left(1+|z|^{2}\right)$ and $A, B$ are constants depending only on $f$. The space $\hat{\mathcal{E}}^{\prime}$ can also be identified as Fourier transforms of distributions with compact support on the real line.

To state our result for $\hat{\mathcal{E}}^{\prime}$ we need the following definition (see [E, p. 523]).

Definition. If $h \in \hat{\mathcal{E}}^{\prime}$, then $h$ is said to be slowly decreasing if there exist constants $\varepsilon>0, A>0$ with the property

(6) $\forall x \in \mathbf{R}, \quad \max \left\{\left|f\left(x^{\prime}\right)\right|: x^{\prime} \in \mathbf{R},\left|x-x^{\prime}\right| \leqslant A \log \left(1+|x|^{2}\right)\right\} \geqslant \varepsilon\left(1+|x|^{2}\right)^{-1 / \varepsilon}$.

THEOREM. If $\left\{z_{k}\right\}_{k=1}^{\infty}=Z(h)$ for $h \in \hat{\mathcal{E}}^{\prime}, h$ slowly decreasing, then $\left\{z_{k}\right\}_{k=1}^{\infty}$ is a universal interpolating sequence for $\hat{\mathcal{E}}^{\prime}$ if and only if

$$
\int_{0}^{\hat{p}\left(z_{k}\right)} n\left(z_{k}, t\right) \frac{d t}{t} \leqslant C \hat{p}\left(z_{k}\right)+D, \quad k=1,2, \ldots,
$$

where $C$ and $D$ are constants independent of $k$.

For the space of functions of finite order $\rho$ and finite type there is a complete description of universal interpolation sequences which is given in $\S 5$.

2. Definitions and notation. We shall always assume that $p(z)$ is a subharmonic function defined for all $z \in \mathbf{C}$, satisfying the following two conditions:

$$
p(z) \geqslant 0 \text { and } \log \left(1+|z|^{2}\right)=O(p(z)) .
$$

There exist constants $K_{1}$ and $K_{2}$ such that $|\zeta-z| \leqslant 1$ implies

$$
p(\zeta) \leqslant K_{1} p(z)+K_{2} \text {. }
$$

Note that (9) says that $p$ is approximately constant on discs of radius 1 .

Definition. $A_{p}=\{f$ entire: $|f(z)| \leqslant A \exp (B p(z))$ for some constants $A, B$ depending only on $f\}$. 
It is easily seen that conditions (8) and (9) on $p(z)$ imply:

All polynomials belong to $A_{p}$.

$$
A_{p} \text { is closed under differentiation, that is, } f \in A_{p} \text { implies } f^{\prime} \in A_{p} \text {. }
$$

The hypotheses on $p$ ensure one can use Hörmander's technique for solving $\partial u / \partial z=w$ with the appropriate growth conditions on $u$ and $w$ (see [BT] for more details).

The two most important examples of such functions $p$ are $p(z)=|z|$ and $\hat{p}(z)=$ $|\operatorname{Im} z|+\log \left(1+|z|^{2}\right)$ corresponding to the space $A_{1}$ of entire functions of exponential type and $\hat{\mathcal{O}}^{\prime}$.

We will now define what we mean by a universal interpolation sequence for the spaces $A_{p}$. Let $V=\left\{\left(z_{k}, m_{k}\right)\right\}_{k=1}^{\infty}$ be a multiplicity sequence, that is, a sequence of points $\left\{z_{k}\right\}_{k=1}^{\infty} \subset \mathbf{C},\left|z_{k}\right| \uparrow \infty$, and a sequence of integers $\left\{m_{k}\right\}_{k=1}^{\infty}$ which correspond to the multiplicities of the points $z_{k}$.

Definition. $A_{p}(V)=\left\{\gamma=\left\{\gamma_{k j}\right\}_{j=0: k=1}^{m_{k}-1: \infty}:\left|\gamma_{k j}\right| \leqslant A \exp \left(B p\left(z_{k}\right)\right)\right.$ for constants $A, B$ independent of $k$ but depending on $\gamma\}$.

With the above definition define the restriction map $\rho: A_{p} \rightarrow A_{p}(V)$ by $\rho(f)=\gamma$ where $f^{(j)}\left(z_{k}\right) / j !=\gamma_{k j}, j=0,1, \ldots, m_{k}-1, k=1,2, \ldots$.

Definition. A multiplicity sequence $V=\left\{\left(z_{k}, m_{k}\right)\right\}_{k=1}^{\infty}$ will be called a universal interpolation sequence for $A_{p}$ if the restriction map $\rho$ is onto.

If $V=\left\{\left(z_{k}, m_{k}\right)\right\}_{k=1}^{\infty}$ is a universal interpolation sequence for $A_{p}$ then we must have $V \subset Z(h)$ for some $h \in A_{p}$. To see this note that $V$ is a universal interpolation sequence implies there exists $g \in A_{p}$ such that $g\left(z_{1}\right)=1, g^{(j)}\left(z_{k}\right) / j !=0, j=0$, $1, \ldots, m_{k}-1, k=2,3, \ldots$. Thus we have $V \subset Z\left(\left(z-z_{1}\right)^{m_{1}} g(z)\right)$ where $g \neq 0$ and $\left(z-z_{1}\right)^{m_{1}} g \in A_{p}$. From now on we will assume $V \subset Z(h)$ for some $h \in A_{p}$.

3. Necessary conditions. Before we state the first theorem some notation is required. Given a space $A_{p}$ and a multiplicity sequence $V=\left\{\left(z_{k}, m_{k}\right)\right\}_{k=1}^{\infty}$, then for constants $L_{1}$ and $L_{2}\left(L_{1} \geqslant K_{1}, L_{2} \geqslant K_{2}\right)$ let $r_{k}$ be the radius of the largest disc centered at $z_{k}$, denoted $D\left(z_{k}, r_{k}\right)$, such that for all $z \in D\left(z_{k}, r_{k}\right), p(z) \leqslant L_{1} p\left(z_{k}\right)+$ $L_{2}$. From condition (9) we see $r_{k} \geqslant 1$.

RemarKs. (i) If $p(z)=|z|^{\rho}, 0<\rho<\infty$, that is, $A_{p}$ is the space of entire functions of order $\rho$ finite type, then $L_{1}$ and $L_{2}$ can be chosen so that $r_{k}=\left|z_{k}\right|$.

(ii) If $A_{p}=\hat{\mathcal{E}}^{\prime}$, then $L_{1}$ and $L_{2}$ can be chosen so that $r_{k} \geqslant \hat{p}\left(z_{k}\right)$.

For a multiplicity sequence $V=\left\{\left(z_{k}, m_{k}\right)\right\}_{k=1}^{\infty}$, define $n\left(t, z_{k}, V\right)$ to be the number of points of $V$, counted with multiplicity, in the punctured disc $D\left(t, z_{k}\right) \sim$ $\left\{z_{k}\right\}$ of radius $t$ centered at $z_{k}$.

THEOREM 1. If $V=\left\{\left(z_{k}, m_{k}\right)\right\}_{k=1}^{\infty}$ is a universal interpolation sequence for $A_{p}$, then the following two conditions hold for constants $C, D$ independent of $k$ :

$$
\begin{gathered}
\int_{0}^{r_{k}} n\left(z_{k}, t, V\right) \frac{d t}{t} \leqslant C p\left(z_{k}\right)+D, \quad k=1,2, \ldots, \\
m_{k} \leqslant \frac{C p\left(z_{k}\right)+D}{\log r_{k}}, \quad k=1,2, \ldots
\end{gathered}
$$


Proof. First we will prove (13). From [S1] we have that if $V$ is a universal interpolation sequence it implies there exists $F \in A_{p}$ such that $V \subset Z(F)$ and $\left(F^{\left(m_{k}\right)} / m_{k} !\right)\left(z_{k}\right)=1$. An application of Cauchy's formula gives

$$
1=\frac{F^{\left(m_{k}\right)}}{m_{k} !}\left(z_{k}\right)=\frac{1}{2 \pi i} \int_{C_{k}} \frac{F(\zeta)}{\left(\zeta-z_{k}\right)^{m_{k}+1}} d \zeta,
$$

where $C_{k}$ is the circle of radius $r_{k}$, center $z_{k}$. Since $F \in A_{p}$ and $p(z) \leqslant L_{1} p\left(z_{k}\right)+L_{2}$ for all $z \in C_{k}$ we have

$$
1 \leqslant \frac{1}{2 \pi} A \exp \left(B p\left(z_{k}\right)\right) \cdot \frac{1}{r_{k}^{m_{k}}} .
$$

From this it follows that $m_{k} \log r_{k} \leqslant C p\left(z_{k}\right)+D$ for constants $C, D$ independent of $k$.

Now we prove equation (12). An application of Jensen's formula in $D\left(z_{k}, r_{k}\right)$ to $F(z) /\left(z-z_{k}\right)^{m_{k}}$ yields

$$
\log \left|\frac{F^{\left(m_{k}\right)}\left(z_{k}\right)}{m_{k} !}\right|+\int_{0}^{r_{k}} n\left(z_{k}, t, Z(F)\right) \frac{d t}{t}=\frac{1}{2 \pi} \int_{0}^{2 \pi} \log \left|F\left(z_{k}+r_{k} e^{i \theta}\right)\right| d \theta-m_{k} \log r_{k} .
$$

Since $F \in A_{p}, r_{k} \geqslant 1$, and $F^{\left(m_{k}\right)}\left(z_{k}\right) / m_{k} !=1$ we have

$$
\int_{0}^{r_{k}} n\left(z_{k}, t, Z(F)\right) \frac{d t}{t} \leqslant C p\left(z_{k}\right)+D
$$

Clearly $n\left(z_{k}, t, V\right) \leqslant n\left(z_{k}, t, Z(F)\right)$ for all $t \geqslant 0$ which implies

$$
\int_{0}^{r_{k}} n\left(z_{k}, t, V\right) \frac{d t}{t} \leqslant C p\left(z_{k}\right)+D
$$

which proves (12).

4. Sufficient conditions. In order to give sufficient conditions for universal interpolation we must put restrictions on the multiplicity sequence $V$. We require the following definition (see [BT, p. 130] for more details).

Definition. A function $f \in A_{p}$ is called slowly decreasing if the following two conditions hold:

(14) There exist $\varepsilon>0, A>0$ such that each connected component $S_{\alpha}$ of the set $S(f, \varepsilon, A)=\{z:|f(z)|<\varepsilon \exp (-A p(z))\}$ is relatively compact.

(15) There exists a constant $B>0$ (independent of $\alpha$ ) such that $p(\zeta) \leqslant B p(z)+B$ for all $\zeta, z \in S_{\alpha}$ and all $\alpha$.

For the case when $p(z)$ is a function of $|z|$ we have the following proposition.

Proposition 1. If $p(z)=p(|z|)$ and $p(2 z)=O(p(z))$, then every $f \in A_{p}, f \neq 0$, is slowly decreasing.

Proof. See [BT, p. 131].

In order to state our sufficient conditions we need an additional restriction on $p(z)$, namely, $p(2 z)=O(p(z))$. We will assume $p$ satisfies this condition for the remainder of this section. 
THEOREM 2. Let $V=\left\{\left(z_{k}, m_{k}\right)\right\}_{k=1}^{\infty}=Z(h)$ for $h \in A_{p}$, h slowly decreasing. If we have

$$
\begin{gathered}
\int_{0}^{r_{k}} n\left(z_{k}, t, V\right) \frac{d t}{t} \leqslant C p\left(z_{k}\right)+D, \\
m_{k} \leqslant \frac{C p\left(z_{k}\right)+D}{\log r_{k}}
\end{gathered}
$$

for constants $C, D$ independent of $k$, then $V$ is a universal interpolation sequence.

Proof. By the appropriate choice of $L_{1}, L_{2}$ in the definition of $r_{k}$ we can assume for all $z_{k}$ that $D\left(z_{k}, r_{k}\right) \cap(S(h, \varepsilon, A))^{c} \neq \varnothing$. Therefore, let $w_{k} \in D\left(z_{k}, r_{k}\right)$ such that $\left|h\left(w_{k}\right)\right| \geqslant \varepsilon \exp \left(-A p\left(z_{k}\right)\right)$. Now apply the minimum modulus theorem to $h$ in the disc $D\left(w_{k}, 2 r_{k}\right)$. For $z \in D\left(w_{k}, 2 r_{k}\right)$, but outside a finite number of excluded circles $\left\{C_{i}\right\}_{i=1}^{n}$ with $\sum_{i=1}^{n}$ radius $\left(C_{i}\right)<\eta r_{k}(\eta<1 / 4)$, we have the estimate

$$
|h(z)| \geqslant \varepsilon^{\prime} \exp \left(-A^{\prime} p\left(z_{k}\right)\right)
$$

where $\varepsilon^{\prime}, A^{\prime}$ do not depend on $k$. Thus there exists a circle centered at $z_{k}$ of radius $\eta_{k} \leqslant r_{k}$ such that for $z \in\left\{\left|z-z_{k}\right|=\eta_{k}\right\}=C\left(z_{k}, \eta_{k}\right)$ we have

$$
|h(z)| \geqslant \varepsilon^{\prime} \exp \left(-A^{\prime} p\left(z_{k}\right)\right) \text {. }
$$

Now apply Jensen's formula to $h(z) /\left(z-z_{k}\right)^{m_{k}}$ in the disc $D\left(z_{k}, \eta_{k}\right)$ which gives us

$$
\log \left|\frac{h^{\left(m_{k}\right)}\left(z_{k}\right)}{m_{k}^{!}}\right|+\int_{0}^{\eta_{k}} n\left(z_{k}, t, V\right) \frac{d t}{t}=\frac{1}{2 \pi} \int_{0}^{2 \pi} \log \left|h\left(z_{k}+\eta_{k} e^{i \theta}\right)\right| d \theta-m_{k} \log \eta_{k} .
$$

Using (16), (17) and the lower bound for $h$ on $C\left(z_{k}, \eta_{k}\right)$, we see

$$
\begin{aligned}
\log \left|\frac{h^{\left(m_{k}\right)}\left(z_{k}\right)}{m_{k} !}\right| \geqslant & -A^{\prime} p\left(z_{k}\right)-\log \frac{1}{\varepsilon^{\prime}}-C p\left(z_{k}\right)-D-C p\left(z_{k}\right)-D, \\
& \log \left|\frac{h^{\left(m_{k}\right)}\left(z_{k}\right)}{m_{k} !}\right| \geqslant-B p\left(z_{k}\right)-B,
\end{aligned}
$$

for some constant $B$ independent of $k$. The lower bound (18) we have obtained implies $V$ is a universal interpolation sequence by a theorem of Berenstein and Taylor (see [BT, p. 124]).

Specializing our results to the case where $A_{p}=\hat{\mathcal{E}}^{\prime}$ we have the following corollary.

COROLlaRY. If $V=\left\{\left(z_{k}, m_{k}\right)\right\}_{k=1}^{\infty}=Z(h)$ for $h \in \hat{\mathcal{E}}^{\prime}, h$ slowly decreasing, then $V$ is a universal interpolation sequence for $\hat{\mathcal{E}}^{\prime}$ if and only if

$$
\begin{gathered}
\int_{0}^{\hat{p}\left(z_{k}\right)} n\left(z_{k}, t, V\right) \frac{d t}{t} \leqslant C \hat{p}\left(z_{k}\right)+D, \\
m_{k} \leqslant \frac{C \hat{p}\left(z_{k}\right)+D}{\log \hat{p}\left(z_{k}\right)},
\end{gathered}
$$

where $C$ and $D$ are constants independent of $k$. 
To have a complete description of universal interpolation sequences for $\hat{\mathcal{E}}^{\prime}$ we still have to answer the following questions.

(i) Since we must always have $V \subset Z(h)$ for $h \in \hat{\mathcal{E}}^{\prime}$, what are the "geometric" conditions which characterize the zero sets of functions in $\hat{\mathcal{E}}^{\prime}$ ?

(ii) Given an arbitrary $V \subset Z(h), h \in \hat{\mathcal{E}}$ ', can we find a "good" description of $V$ in terms of slowly decreasing functions in $\hat{\mathcal{E}}^{\prime}$ ?

In the next section we will answer question (ii) for functions of finite order and thus obtain a complete description of universal interpolation sequences for these spaces.

5. Universal interpolation for functions of finite order. We will denote the space of entire functions of order $\rho$ and finite type by $A_{\rho}$, that is, $A_{p}=A_{\rho}$ where $p(z)=|z|^{\rho}$. For the spaces $A_{\rho}$ we have the following interpolation theorem.

TheOREM 3. If $V=\left\{\left(z_{k}, m_{k}\right)\right\}_{k=1}^{\infty} \subset Z(h)$ for some $h \in A_{\rho}$, then $V$ is a universal interpolation sequence for $A_{\rho}$ if and only if there exist constants $C, D$ independent of $k$ such that

$$
\begin{gathered}
\int_{0}^{\left|z_{k}\right|} n\left(z_{k}, t, V\right) \frac{d t}{t} \leqslant C\left|z_{k}\right|^{\rho}+D, \\
m_{k} \leqslant \frac{C\left|z_{k}\right|^{\rho}+D}{\log \left|z_{k}\right|} .
\end{gathered}
$$

Remark. Condition (19') implies

(21) for all $k$, there exist constants $C$ and $D$ such that

$$
n\left(z_{k}, t, V\right) \leqslant \frac{C\left|z_{k}\right|^{\hat{\rho}}+D}{\log \left|z_{k}\right|-\log t} \quad \text { for } 0 \leqslant t \leqslant\left|z_{k}\right| \text {. }
$$

ProOF.

$$
n\left(z_{k}, t, V\right)\left[\log \left|z_{k}\right|-\log t\right] \leqslant \int_{0}^{\left|z_{k}\right|} n\left(z_{k}, t, V\right) \frac{d t}{t} \leqslant C\left|z_{k}\right|^{\rho}+D .
$$

Therefore

$$
n\left(z_{k}, t, V\right) \leqslant \frac{C\left|z_{k}\right|^{p}+D}{\log \left|z_{k}\right|-\log t} \quad \text { for } 0 \leqslant t \leqslant\left|z_{k}\right|
$$

Later in this section we will give an example to show that $\left(19^{\prime}\right)$ and (21) are not equivalent.

Proof OF TheOrem 3. $(\rightarrow)$ The proof of necessity is just a special case of Theorem 1.

$(\Leftarrow)$ Sufficiency in the case when $\rho$ is not an integer follows from Theorem 2 since it is well known that $V=Z(h)$ for some $h \in A_{\rho}$ and $h$ is slowly decreasing by Proposition 1. Thus we need only consider the case when $\rho$ is an integer. For this case we need a "good" description of $V$ which is provided by the following Lemma.

LEMMA. If $V=\left\{\left(z_{k}, m_{k}\right)\right\}_{k=1}^{\infty} \subset Z(h), h \in A_{\rho}$, $\rho$ an integer, then there exists a function $f \in A_{\rho}$ such that $V \subset Z(f)$ and there exists a family of discs $\left\{D_{i}\right\}_{i=1}^{\infty}$ which satisfy (22)-(25) below. 
(22) There exist constants $\varepsilon, C$ such that

$$
|f(z)| \geqslant \varepsilon \exp \left(-C|z|^{\rho}\right) \quad \text { for } z \in \mathbf{C} \sim \bigcup_{i=1}^{\infty} D_{i} .
$$

(23) Each $D_{i}$ contains either points of $V$ or points of $Z(f) \sim V$ but not both.

(24) $\Sigma_{D_{i} \subset\{z \mid \leqslant r\}}$ radius $\left(D_{i}\right)<\eta r$ for some fixed constant $\eta<1 / 4$.

(25) If $\rho$ is an odd integer the points of $Z(f) \sim V$ form a subsequence of $\left\{(\xi n)^{1 / \rho}\right\}_{n=-\infty}^{\infty} \cup\left\{i(\xi n)^{1 / \rho}\right\}_{n=-\infty}^{\infty}$ for some fixed constant $\xi$. If $\rho$ is an even integer the points of $Z(f) \sim V$ form a subsequence of $\left\{(\xi n)^{1 / \rho} e^{i \pi / \rho}\right\}_{n=0}^{\infty} \cup\left\{(\xi n)^{1 / \rho}\right\}_{n=0}^{\infty} \cup$ $\left\{(\xi n)^{1 / \rho} e^{i \pi / 2 \rho}\right\}_{n=0}^{\infty} \cup\left\{(\xi n)^{1 / \rho} e^{i 3 \pi / 2 \rho}\right\}_{n=0}^{\infty}$ for some fixed constant $\xi$.

Proof. For simplicity we will prove the lemma in the case that $\rho=1$ and $m_{k}=1$, $k=1,2, \ldots$. The proof of the general case uses the same ideas; however, the technical details are more difficult.

The basic idea behind the construction of $f$ is to add balancing zeros $V^{\prime}$ to $V$ and define $f$ to be the canonical product with zero set $V \cup V^{\prime}$. That is, we define $f$ as

$$
f(z)=\prod_{W_{h} \in V \cup V^{\prime}}\left(1-\frac{z}{W_{k}}\right) e^{z / W_{k}} .
$$

In order that $f$ be of exponential type, the balancing zeros $V^{\prime}$ must be chosen sufficiently distant from $V$ so that Lindelöf's condition holds, namely,

$$
\begin{aligned}
& \left|\operatorname{Re}\left\{\sum_{|=k| \leqslant r} \frac{1}{z_{k}}+\sum_{\substack{\vec{\theta}_{k}^{\prime} \in V^{\prime} \\
\left|\hat{\theta}_{k}\right| \leqslant r}} \frac{1}{\mathbb{Q}_{k}}\right\}\right| \leqslant K, \\
& \left|\operatorname{Im}\left\{\sum_{|=k| \leqslant r} \frac{1}{z_{k}}+\sum_{\substack{\mathbb{Q}_{k} \in \in V^{\prime} \\
\left|\hat{Q}_{k}^{\prime}\right| \leqslant r}} \frac{1}{\mathbb{Q}_{k}}\right\}\right| \leqslant K
\end{aligned}
$$

for some constant $K$ independent of $r$. Of course we must also choose $V^{\prime}$ in order that (22)-(25) are satisfied.

It will be shown that it is possible to place the balancing zeros $V^{\prime}$ on the real and imaginary axes. The procedure for placing the balancing zeros does not depend on which axis is chosen so we will only consider the case where we are adding balancing zeros on the real axis to satisfy (26).

For the remainder of the proof we will use the following notation: $A_{n}=\left\{z \mid 2^{n-1}\right.$ $\left.\leqslant|z|<2^{n}\right\}, V_{n}=A_{n} \cap V=\left\{z_{k}^{n}\right\}_{k^{n}=1}^{\lambda_{n}}, D(z, r)=\operatorname{disc}$ of radius $r$, center $z$ and $R(D)$ = radius of the disc $D$.

The first step is to construct the discs about the points of $V$. We will then place the balancing zeros $V^{\prime}$ sufficiently distant from these discs in order to satisfy (22) and (23). Apply the proof of the Boutroux-Cartan lemma (see [Le, p. 19] for a proof) to determine the discs about the zeros in $V_{n}$. That is, construct discs $D_{i}^{n}, i=1, \ldots, i_{n}$, about the points of $V_{n}$ such that $\sum_{i=1}^{i_{n}} R\left(D_{i}^{n}\right)=2 \eta^{\prime} 2^{n}$ for some fixed constant $\eta^{\prime}$ to be determined later. The discs $D_{i}^{n}$ have the property that the number of points of $V_{n}$ inside $D_{i}^{n}$ is equal to $\left(\eta^{\prime} 2^{n} / \lambda_{n}\right) R\left(D_{i}^{n}\right)$, where $\lambda_{n}$ is the number of points in $V_{n}$. To 
simplify notation let $\eta^{\prime} 2^{n} / \lambda_{n}=\delta_{n}$. The important property of the discs $D_{i}^{n}$ is that for any $z \notin \cup_{i=1}^{i_{n}} D_{i}^{n}$, a disc of radius $k \delta_{n}$ ( $k$ an integer) about $z$ contains at most $k-1$ points of $V_{n}$.

Increase the radius of the discs $D_{i}^{n}$ by $3 \delta_{n}$ and denote the new discs by $\tilde{D}_{i}^{n}$. The discs $\tilde{D}_{i}^{n}$ have the property that for any $z \notin \cup_{i=1}^{i_{n}} \tilde{D}_{i}^{n}, D\left(z, 2 \delta_{n}\right)$ is at least a distance $\delta_{n}$ away from any of the discs $D_{i}^{n}$. Note that since there are at most $\lambda_{n}$ discs we have

$$
\sum_{i=1}^{i_{n}} R\left(\tilde{D}_{i}^{n}\right) \leqslant 5 \eta^{\prime} 2^{n}
$$

In future we will call the discs $\tilde{D}_{i}^{n}$ " omitted discs."

Since the balancing zeros must not lie in the interior of any of the discs $\tilde{D}_{i}^{n}$, we must determine how much of the real axis remains in which to place the balancing zeros. The portion of the real axis in $A_{n} \cap\left(\cup_{i=1}^{i_{n}} \tilde{D}_{i}^{n}\right)$ can come from three sources:

(i) Omitted discs about points in $V_{n}$-from (28) this can be no more than $5 \eta^{\prime} 2^{n}$.

(ii) Omitted discs about points in $V_{n-1}$ - no more than two discs and thus the portion of the real axis which these two discs cover is no more than $2 \eta^{\prime} 2^{n-1}=\eta^{\prime} 2^{n}$.

(iii) Omitted discs about points in $V_{n+1}$-at most $2 \eta^{\prime} 2^{n+1}=4 \eta^{\prime} 2^{n}$. Thus the total length of $\mathbf{R} \cap A_{n}$ covered by the omitted discs can be at most $10 \eta^{\prime} 2^{n}$.

The next step is to determine the placing of the balancing zeros. We will show that the balancing zeros can be chosen as a subsequence of $\{(\xi j)\}_{j=-\infty}^{\infty}$ where $\xi$ is a fixed constant. This will ensure that (25) holds.

It will be convenient to choose $\xi$ in order that the distance, $\xi$, between points of $\{(\xi j)\}_{j=-\infty}^{\infty}$, is less than or equal to $\delta_{n}$ for all $n$. A lower bound for $\delta_{n}$ is easily computed as follows:

$$
\delta_{n}=\frac{\eta^{\prime} 2^{n}}{\lambda_{n}} \geqslant \frac{\eta^{\prime} 2^{n}}{C 2^{n}}=\frac{\eta^{\prime}}{C}
$$

since $\lambda_{n} \leqslant n\left(V, 2^{n}\right) \leqslant C 2^{n}$ for all $n$. Thus we can choose $\xi=\eta^{\prime} / C$.

We wish to choose a subsequence $\left\{\mathbb{Q}_{j}^{n}\right\}_{j=1}^{j_{n}}$ of $\{(\xi j)\}_{j=-\infty}^{\infty} \cap A_{n}$ such that the $\mathbb{Q}_{j}^{n}$ lie outside the omitted discs and the following two properties hold:

The distance between any two balancing zeros is at least $16 \delta_{n}$.

$$
\left|\sum_{j=1}^{j_{n}} \frac{1}{\mathbb{Q}_{j}^{n}}+\operatorname{Re}\left\{\sum_{z_{k} \in V_{n}} \frac{1}{z_{k}}\right\}\right|<\frac{1}{2^{n-1}} .
$$

If we assume (29) for the moment, then the discs $D_{i}$ will be the union of $D\left(Q_{j}^{n}, 2 \delta_{n}\right)$, $j=1, \ldots, j_{n}, n=1,2, \ldots$, and $D_{i}^{n}, i=1, \ldots, i_{n}, n=1,2, \ldots$ By construction these discs satisfy (23) since each $D\left(\mathbb{Q}_{j}^{n}, 2 \delta_{n}\right)$ is at least a distance $\delta_{n}$ from any of the discs $D_{i}^{n}$. From (29) it is also clear that (24) holds since $\sum_{\left|\mathcal{Q}_{j}^{n}\right| \leqslant r} R\left(D\left(\mathbb{Q}_{j}^{n}, 2 \delta_{n}\right)\right) \leqslant r / 8$ and for $\eta^{\prime}<1 / 8$ we have $\Sigma_{D_{i}^{n} \subset\{k \mid<r\}} R\left(D_{i}^{n}\right)<r / 8$ which together imply $\Sigma_{D_{i} \subset\{z \mid<r\}} R\left(D_{i}\right)$ $<\eta r$ for some $\eta<1 / 4$.

In order to show that a subsequence $\left\{\mathbb{Q}_{j}^{n}\right\}_{j=1}^{j_{n}}$ can be chosen to satisfy (30) we will only consider the worst possible case. This is when the omitted discs cover as many points of $\{(\xi j)\}_{j=-\infty}^{\infty}$ as possible, starting at $2^{n-1}$ and proceeding consecutively. The 
smallest value of $\operatorname{Re}\left\{\sum_{z_{k} \in V_{n}}\left(1 / z_{k}\right)\right\}$ occurs when all $z_{k}$ are clustered at $-2^{n-1}$ so we see

$$
\operatorname{Re}\left\{\sum_{z_{k} \in V_{n}} \frac{1}{z_{k}}\right\} \geqslant-\frac{\lambda_{n}}{2^{n-1}} .
$$

If we can find the subsequence $\left\{\mathbb{Q}_{j}^{n}\right\}_{j=1}^{j_{n}}$ satisfying (30) for this case, then for any other case, $\sum_{j=1}^{j_{n}}\left(1 / \mathbb{Q}_{j}^{n}\right)$ can be chosen at least as positive, while $\operatorname{Re}\left\{\sum_{z_{k} \in V_{n}}\left(1 / z_{k}\right)\right\}$ can be no more negative.

First let us choose $m$ so that $20 \delta_{n}>m \xi>16 \delta_{n}$, which can be done since by the choice of $\xi, \xi \leqslant \delta_{n}$. Thus, if we choose $\left\{\mathcal{Q}_{j}^{n}\right\}_{j=1}^{j_{n}} \subset\{(\xi m j)\}_{j=-\infty}^{\infty} \cap A_{n}$, then $m \xi$ is the minimum distance between any two points of $\left\{\mathbb{Q}_{j}^{n}\right\}_{j=1}^{j_{n}}$ and (29) is satisfied.

To show (30) holds, the following Proposition will be needed.

Proposition. Let

$$
I_{n}=\left(a_{n}, b_{n}\right) \subset(a, b), \quad a>0, n=1,2, \ldots, N,
$$

with $\sum_{n=1}^{N}\left(b_{n}-a_{n}\right)=L$ and let $\sigma=\min \left(b_{n}-a_{n}\right)$. Consider $\{(\zeta j)\}_{j=1}^{\infty} \cap((a, b) \sim$ $\left.\cup_{i j=1}^{N} I_{n}\right)=\left\{\left(\zeta j_{l}\right)\right\}_{l=1}^{M}$, that is, all points of the sequence in any of the $I_{n}$. With the above notation we have

$$
\sum_{l=1}^{M} \frac{1}{\zeta j_{l}} \geqslant \frac{1}{2 \zeta} \log \left[\frac{b}{a+(\zeta / \sigma+1) L}\right]
$$

Proof. The minimum value of the sum occurs when the intervals $I_{n}$ cover all points of the sequence $\{(\zeta j)\}_{j=1}^{\infty}$ starting at $a$ and proceeding consecutively. Since the lengths of the intervals may be less than $\zeta$, the distance between two points of the sequence, more points could be covered than just those in the interval $(a, a+L)$. To determine how many more points could be covered, note that at most the points in the interval $(a, a+(\zeta / \sigma+1) L)$ can be covered by the intervals $I_{n}$.

Thus the minimum value of the sum is

$$
\sum_{l=1}^{M} \frac{1}{\zeta j_{l}} \geqslant \sum_{\zeta j \in(a+(\zeta / \sigma+1) L, b)} \frac{1}{\zeta j} \geqslant \frac{1}{2 \zeta} \log \left[\frac{b}{a+(\zeta / \sigma+1) L}\right] .
$$

This concludes the proof of the Proposition.

Now apply the Proposition to the case where the omitted discs intersect as large a length as possible of the interval $\left(2^{n-1}, 2^{n}\right)$. Thus we have $a=2^{n-1}, b=2^{n}$, $\sigma=16 \delta_{n}, \zeta=m \xi, L=10 \eta^{\prime} 2^{n}$. With the above substitutions, the Proposition gives us

$$
\begin{aligned}
\sum_{j=1}^{j_{n}} \frac{1}{Q_{j}^{n}} & \geqslant \frac{1}{2 m \xi} \log \left[\frac{2^{n}}{2^{n-1}+\left(m \xi / 16 \delta_{n}+1\right) 10 \eta^{\prime} 2^{n}}\right] \\
& \geqslant \frac{1}{2 m \xi} \log \left[\frac{2}{1+\left(m \xi / 16 \delta_{n}+1\right) 20 \eta^{\prime}}\right] \\
& \geqslant \frac{1}{2 m \xi} \log \left[\frac{2}{1+\eta^{\prime \prime}}\right]
\end{aligned}
$$

where $\eta^{\prime \prime}=\left(m \xi / 16 \delta_{n}+1\right) 20 \eta^{\prime}$. 
Since we have chosen $m$ so that $20 \delta_{n}>m \xi$, we have

$$
\sum_{j=1}^{j_{n}} \frac{1}{\mathbb{Q}_{j}^{n}} \geqslant \frac{1}{40 \delta_{n}} \log \left[\frac{2}{1+\eta^{\prime \prime}}\right] .
$$

In order for the balancing zeros to cancel $-\lambda_{n} / 2^{n-1}$, the following inequality is sufficient:

$$
\frac{1}{40 \delta_{n}} \log \left[\frac{2}{1+\eta^{\prime \prime}}\right] \geqslant \frac{\lambda_{n}}{2^{n-1}},
$$

or equivalently (using the fact that $\lambda_{n}=\eta^{\prime} 2^{n} / \delta_{n}$ ),

$$
\frac{1}{40} \log \left[\frac{2}{1+\eta^{\prime \prime}}\right] \geqslant 2 \eta^{\prime} \text {. }
$$

The LHS of the above inequality will be bounded below for $\eta^{\prime}$ sufficiently small since $\eta^{\prime} \rightarrow 0$ implies $\eta^{\prime \prime} \rightarrow 0$. Thus there is some positive value of $\eta^{\prime}$ for which the inequality holds (for all $n$ ).

Property (30) is now satisfied for the appropriate choice of subsequence $\left\{\mathbb{Q}_{j}^{n}\right\}_{j=1}^{j_{n}}$ since we add just enough balancing zeros $\mathbb{Q}_{j}^{n}$ to make the error as small as possible. The preceding argument has shown that enough balancing zeros can be added to make the error no worse than the reciprocal of the largest term in $\left\{Q_{j}^{n}\right\}_{j=1}^{j_{n}}$, which is $\leqslant 1 / 2^{n-1}$. This proves that the function $f$ is of exponential type.

Finally, the proof of (22) is a variant of the proof of the minimum modulus theorem for functions of exponential type (see [Le, p. 21]). First, to simplify notation, let

$$
W_{n}=\left(\bigcup_{k=1}^{n} V_{k}\right) \cup\left(\bigcup_{k=1}^{n}\left\{\mathbb{Q}_{j}^{k}\right\}_{j=1}^{j_{n}}\right)
$$

It will suffice to show, for $z \in A_{n-1} \cup A_{n}$, the polynomial $P_{n}(z)=\prod_{w_{k} \in w_{n}}\left(z-w_{k}\right)$ satisfies, outside the omitted discs and outside the discs $D\left(\mathbb{Q}_{j}^{k}, 2 \delta_{k}\right), j=1, \ldots, j_{k}$, $k=1, \ldots, n$, the bound $\left|P_{n}(z)\right|>\left(\eta^{\prime} 2^{n-3} / e\right)^{P_{n}}$, where $p_{n}=\sum_{k=1}^{n}\left(\lambda_{k}+j_{k}\right)$.

Let $V_{k}^{\prime}=V_{k} \cup\left\{\mathbb{Q}_{j}^{k}\right\}_{j=1}^{j_{k}}$ and let $q_{k}=\lambda_{k}+j_{k}$ be the number of zeros in $V_{k}^{\prime}$. Choose $z \in A_{n-1} \cup A_{n}, z$ outside the omitted discs and the discs $D\left(\mathbb{Q}_{j}^{k}, 2 \delta_{k}\right)$, and order the zeros of $V_{k}^{\prime}$ in order of increasing distance from $z$ and label the zeros $\left\{\gamma_{l}^{k}\right\}_{l=1}{ }_{k}$ in the new ordering.

Claim. For any $k \leqslant n,\left|z-\gamma_{l}^{k}\right|>l\left(\delta_{k} / 2\right), l=1, \ldots, q_{k}$.

Proof. The claim is equivalent to the statement that a disc of radius $N\left(\delta_{k} / 2\right)$ contains at most $N-1$ zeros of $V_{k}^{\prime}$. Since $z$ is at least a distance $2 \delta_{k}$ from all zeros of $V_{k}^{\prime}$, the statement is true for $N \leqslant 4$. For $N>4$, a disc $D\left(z, N \delta_{k} / 2\right)$ contains at most $(N+1) / 2-2$ zeros of $V_{k}$ and at most $N / 16+1$ zeros of the balancing zeros $\left\{Q_{j}^{k}\right\}_{j=1}^{j_{k}}$. This gives a total of $7 N / 16-1 / 2$ zeros of $V_{k}^{\prime}$ which is less than $N-1$ for $N>4$. This implies the claim. 
Consider the polynomials $Q_{k}(z)=\prod_{l=1}^{q_{k}}\left(z-\gamma_{l}^{k}\right)$. Application of the claim gives us the following lower bound for $Q_{k}(z)$ :

$$
\begin{aligned}
\left|Q_{k}(z)\right| & >\prod_{l=1}^{q_{k}} l\left(\frac{\boldsymbol{\delta}_{k}}{2}\right), \quad k=1,2, \ldots, n, \\
& >\left(q_{k} !\right)\left(\frac{\eta^{\prime} 2^{k}}{2 \lambda_{k}}\right)^{q_{k}}>\left(\frac{\eta^{\prime} 2^{k-1}}{e}\right)^{q_{k}} .
\end{aligned}
$$

Using the above lower bound for $k=n, n-1, n-2$ and noting that the distance from $z \in A_{n} \cup A_{n-1}$ to any zero in $D_{n-3}=\left\{z|| z \mid<2^{n-3}\right\}$ is at least $2^{n-3}$, we have the lower bound $\left|P_{n}(z)\right|>\left(\eta^{\prime} 2^{n-3} / e\right)^{P_{n}}$.

With the estimate for $P_{n}(z)$ computed above, the proof of (22) is precisely the proof of the minimum modulus theorem for functions of exponential type. This concludes the proof of the Lemma.

As in the proof of Theorem 2 it will suffice to prove

$$
\left|\frac{f^{\left(m_{k}\right)}\left(z_{k}\right)}{m_{k} !}\right| \geqslant \varepsilon \exp \left(-A\left|z_{k}\right|^{\rho}\right) \quad \text { for } k=1,2, \ldots,
$$

for constants $\varepsilon, A$ independent of $k$. By (22) and (24) we can find a circle $C_{k}\left(z_{k}, r_{k}\right)$ with $r_{k} \leqslant\left|z_{k}\right|$ such that $|f(z)| \geqslant \varepsilon \exp \left(-C\left|z_{k}\right|^{\rho}\right)$ for all $z \in C\left(z_{k}, r_{k}\right)$. As before we wish to apply Jensen's formula to $f(z) /\left(z-z_{k}\right)^{m_{k}}$ in the region bounded by $C\left(z_{k}, r_{k}\right)$. However, $C\left(z_{k}, r_{k}\right)$ contains zeros of $f$ which are not in $V$ so we must use (23) and (25) to obtain an estimate of $\int_{0}^{r_{k}} n\left(z_{k}, t, Z(f) \sim V\right) d t / t$. Using (25) we see that $n\left(z_{k}, t, Z(f) \sim V\right) \leqslant K t^{\rho}$ for all $t$ where $K$ is a constant depending only on $\xi$. Thus we have

$$
\int_{0}^{r_{k}} n\left(z_{k}, t, Z(f) \sim V\right) \frac{d t}{t} \leqslant C r_{k}^{\rho}+D, \quad k=1,2, \ldots,
$$

where $C$ and $D$ are constants independent of $k$. From Jensen's formula we obtain

$$
\log \left|\frac{f^{\left(m_{k}\right)}\left(z_{k}\right)}{m_{h} !}\right|+\int_{0}^{r_{k}} n\left(z_{k}, t, Z(f)\right) \frac{d t}{t}=\frac{1}{2 \pi} \int_{0}^{2 \pi} \log \left|f\left(z_{k}+r_{k} e^{i \theta}\right)\right| d \theta-m_{k} \log r_{k} .
$$

Therefore we have

$$
\log \left|\frac{f^{\left(m_{k}\right)}\left(z_{k}\right)}{m_{k} !}\right| \geqslant-A r_{k}^{\rho}-\log \frac{1}{\varepsilon}-C r_{k}^{\rho}-D-\int_{0}^{r_{k}} n\left(z_{k}, t, Z(f)\right) \frac{d t}{t} .
$$

From $\left(19^{\prime}\right)$ and (32) we see

$$
\begin{aligned}
\int_{0}^{r_{k}} n\left(z_{k}, t, Z(f)\right) \frac{d t}{t} & =\int_{0}^{r_{k}} n\left(z_{k}, t, V\right) \frac{d t}{t}+\int_{0}^{r_{k}} n\left(z_{k}, t, Z(f) \sim V\right) \frac{d t}{t} \\
& \leqslant C\left|z_{k}\right|^{\rho}+D
\end{aligned}
$$

and thus we have

$$
\log \left|\frac{f^{\left(m_{k}\right)}\left(z_{k}\right)}{m_{k} !}\right| \geqslant-C\left|z_{k}\right|^{\rho}-D
$$


for constants $C, D$ independent of $k$. This proves (31) and thus we can conclude $V$ is a universal interpolation sequence for $A_{\rho}$. This completes the proof of Theorem 3 .

We will now give an example to show conditions $\left(19^{\prime}\right)$ and $(21)$ are not equivalent.

Example. We will construct an even function $f$ of exponential type such that $V=Z(f)$ and $V$ satisfies condition (21) but not $\left(19^{\prime}\right)$.

Consider the functions $f_{n}(r)=2^{2 n} /\left(\log 2^{2 n}-\log r\right), 0 \leqslant r \leqslant 2^{2 n-1} / e^{2}=C_{n}$. Place zeros on the real axis from $2^{2 n}$ to $2^{2 n}+C_{n}$ such that $n\left(2^{2 n}, r\right)=\left[f_{n}(r)\right]$, $0 \leqslant r \leqslant C_{n}$ (where [ ] denotes the greatest integer function). We will also assume that there is a zero of multiplicity one at $2^{2 n}$ for all $n$. Let $V_{n}$ denote the zeros in the interval $\left[2^{2 n}, 2^{2 n}+C_{n}\right]$ and let $V=\cup_{n=1}^{\infty}\left(V_{n} \cup\left(-V_{n}\right)\right)$ where $-V_{n}$ is $V_{n}$ reflected about 0 . It is clear that $V=Z(f)$ for some even function $f$ of exponential type.

Claim 1. Condition (21) holds for the sequence $V$, namely,

$$
n\left(z_{k}, r, V\right) \leqslant \frac{C\left|z_{k}\right|+D}{\log \left|z_{k}\right|-\log r}, \quad z_{k} \in V, 0 \leqslant r \leqslant\left|z_{k}\right| .
$$

Since $V$ is the zero set of a function of exponential type it is sufficient to prove (21) for $0 \leqslant r \leqslant\left|z_{k}\right| / 2$. For convenience, let $N=\left[C_{n}\right], a_{0}=2^{2 n}$ and let $\left\{a_{k}\right\}_{k=0}^{N}=V_{n}$ with $a_{k}<a_{k+1}$. If $a_{k}=a_{0}$ then condition (21) holds from the construction of $V_{n}$. We must therefore show (21) holds for all $a_{k} \in V_{n}, a_{k} \neq a_{0}$. Note that $n\left(a_{k},\left|a_{k}\right| / 2, V\right)$ will only include zeros of $V_{n}$ for any $a_{k} \in V_{n}$.

A simple computation shows that

$$
a_{k+1}-a_{k}>a_{l+1}-a_{l} \text { for } 0 \leqslant l \leqslant k \leqslant N,
$$

that is, the distance between two consecutive zeros increases as $k$ increases. Beginning at $a_{0}$, the distance between two consecutive zeros must be increasing and therefore for any other zero $a_{k} \in V_{n}$, we must have

$$
n\left(a_{k}, r, V\right) \leqslant \frac{2 a_{0}}{\log a_{0}-\log r} \leqslant \frac{3 a_{k}}{\log a_{k}-\log r}, \quad 0 \leqslant r \leqslant C_{n} .
$$

Thus condition (21) holds for the sequence $V$.

Claim 2. Condition (19') fails for $V$.

First, it is easy to see that

$$
n\left(a_{0}, r, V\right) \geqslant \frac{a_{0}}{2\left(\log a_{0}-\log r\right)} \text { for } \eta \leqslant r \leqslant C_{n},
$$

where $\eta$ is chosen so that $n\left(a_{0}, \eta, V\right)=1$. Thus we have the inequality

$$
\int_{\eta}^{C_{n}} n\left(a_{0}, t, V\right) \frac{d t}{t} \geqslant \frac{1}{2} \int_{\eta}^{C_{n}} \frac{a_{0}}{\left(\log a_{0}-\log t\right)} \frac{d t}{t} \geqslant \frac{a_{0}}{4} \log \log a_{0},
$$

since $\eta<1$ and $C_{n}=a_{0} / 2 e^{2}$. Therefore,

$$
\int_{\eta}^{C_{n}} n\left(a_{0}, t, V\right) \frac{d t}{t} \geqslant 2^{2 n-2} \log \log 2^{2 n},
$$

and clearly there do not exist any positive constants $C$ and $D$, such that

$$
\int_{0}^{2^{2 n-1}} n\left(2^{2 n}, t, V\right) \frac{d t}{t} \leqslant C 2^{2 n}+D
$$

Conclude that $\left(19^{\prime}\right)$ fails for $V$. Thus $V$ is the required counterexample. 


\section{REFERENCES}

[BT] C. A. Berenstein and B. A. Taylor, A new look at interpolation theory for entire functions of one variable, Adv. in Math. 33 (1979), 109-143.

[E] L. Ehrenpreis, Solutions of some problems of division. IV, Amer. J. Math. 57 (1960), 522-588.

[L] A. F. Leont'ev, Representation of functions by generalized Dirichlet series, Math. U.S.S.R. Izv. 6 (1972), 1265-1277.

[Le] B. Ja. Levin, Distribution of zeros of entire functions, Transl. Math. Monos., Vol. 5, Amer. Math. Soc., Providence, R.I., 1964.

[S1] W. A. Squires, Necessary conditions for universal interpolation in है', Canad. J. Math. 33 (1981), 1356-1364.

[S2] Ph.D. Thesis, University of Michigan, 1979.

Department of Mathematics, California institute of Technology, Pasadena, California 91125 